\title{
Trypanosomiasis in a Young Infant from Rural Gujarat, India
}

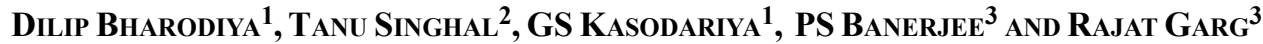 \\ From ${ }^{1}$ Department of Pediatrics, Apple Hospital, Surat; ${ }^{2}$ Kokilaben Dhirubhai Ambani Hospital and Medical Research Institute, \\ Andheri West, Mumbai; and ${ }^{3}$ ICAR-Indian Veterinary Research Institute, Izatnagar, UP; India.
}

Correspondence to:

Dr. Tanu Singhal,

Department of Pediatrics, Kokilaben

Dhirubhai Ambani Hospital and Medical

Research Institute, Andheri West, Mumbai,

India.tanusinghal@yahoo.com

Received: December 25, 2016;

Initial review: December 27, 2016;

Accepted: October 10, 2017.

\begin{abstract}
Background: Human trypansomiasis due to infection by animal trypanosomes is rarely reported from India. Case characteristics: We describe clinical presentation of a 2-monthold boyfrom a rat infested house in rural Gujarat who was diagnosed to be havinginfection with the rodent parasite Trypanosoma lewisi. Observation: The fever and parasitemia resolved on treatment with liposomal amphotericin B, Ceftriaxone and Amikacin, and there was no recurrence of parasitemia over a 2 month follow-up. Message: The case highlights the need for increased awareness and heightened surveillance for this rare zoonotic infection.
\end{abstract}

Keywords: Protozoa, Pyrexia of unknown origin, T. Lewisi.

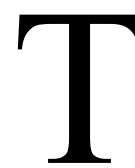

rypanosomes are affecting humans

flagellated protozoans and animals. Human trypanosomiasis is endemic in Africa as sleeping sickness (caused by Trypanosoma brucei) and Latin America as Chagas disease (caused by T. cruzi). Human trypanosomiasis is not known to exist in India. However, animal trypanosomiasis is endemic in India, and is caused by either T. evansi in cattle/ horses (causing a disease called "surra") or T. lewisi in rats [1]. Only sporadic cases of human infection with animal trypanosomes have been reported from India. We report the case of a young infant who had T. lewisi infection.

\section{Case Report}

A 2-month-old infant born to farmer parents of lowermiddle socioeconomic status in Dhari village of Amreli district of Gujarat presented to a private hospital in Surat with history of fever of 5 days duration. The mother reported an insect/flea bite (common in cattle and termed as "itardi" in local language) in the infant's axilla about 2 weeks prior to this episode of fever.The insect was removed following which there was some local bleeding but thereafter the baby was asymptomatic. There was a history of the house being infested by rats. At presentation, the baby was playful and active but had pallor and hepatomegaly. Routine investigations revealed hemoglobin $(\mathrm{Hb})$ of $8.3 \mathrm{~g} / \mathrm{dL}$, total leucocyte count of $10.5 \times 10^{9} / \mathrm{L}$, platelet count of $130 \times 10^{9} / \mathrm{L}$ and an elevated ALT (582 IU/1). A peripheral smear, sent for malarial parasite, incidentally showed flagellated parasites suggestive of Trypanosoma (Fig. 1). Blood samples, peripheral smears and filter paper samples were sent to the ICAR - Indian Veterinary Research Institute (IVRI) at Izatnagar, UP for confirmation and identification.

The child was hospitalized, blood cultures were sent, and therapy with ceftriaxone, amikacinand liposomal amphotericin B was initiated. This treatment was for presumed bacterial sepsis and trypanosomiasis based on experience from a previous similar case reported from India [2]. We could not procure pentamidine and suramin. The baby was followed up clinically and with daily blood counts, Alanine aminotransferase (ALT) and

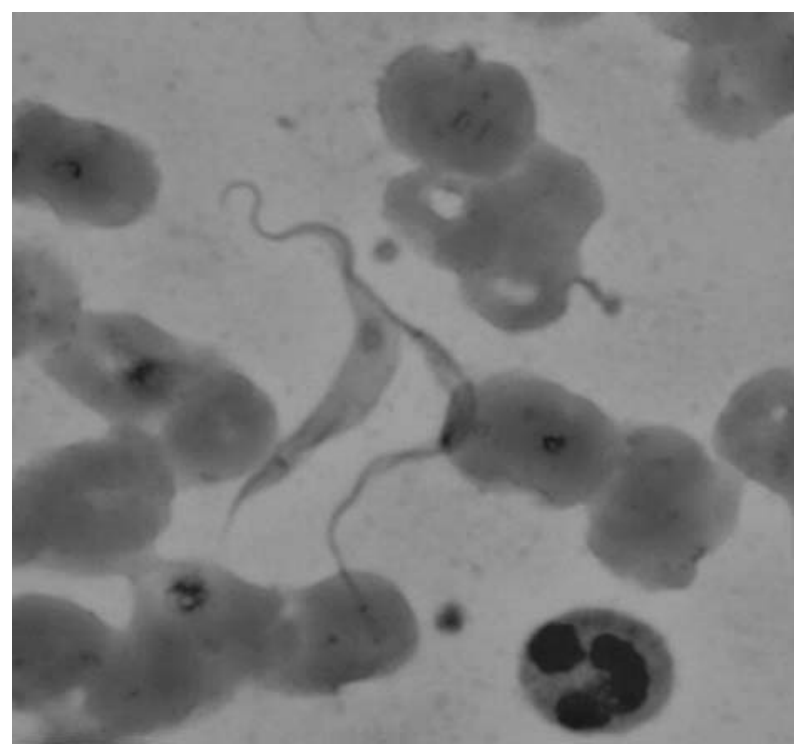

FIG.1 Peripheral smear showing flagellated parasites with subterminal kinetoplast resembling trypanosoma. 
peripheral smear. Over the next five days, the hemoglobin dropped from $8.3 \mathrm{~g} / \mathrm{dL}$ to $6.9 \mathrm{~g} / \mathrm{dL}$ but platelet count increased to $522 \times 10^{9} / \mathrm{L}$. The ALT levels increased to 1120 IU/L. The peripheral smear showed reduction in parasitemia from 3-4 parasites per high power field to 1 parasite per 3-4 fields by day 3 and no parasites by day 5 of treatment. Antimicrobials were stopped after 5 days and the baby was discharged. A week later, the liver enzymes had normalized and there was sustained absence of parasites. The child was well over the next 2 month followup period with no reappearance of parasitemia. The hemoflagellate in the smear was identified as T. lewisi on the basis of morphology and PCR amplification of ITS1 region with flanking $18 \mathrm{~S}$ and 5.8S rRNA(Web Fig.1).

\section{Discussion}

The clinical differentials in this young febrile infant were bacterial sepsis, malaria or viral fever. The diagnosis of trypanosomiasis was unanticipated, surprising and incidental as this parasitic infection in humans is very rare in India. The characteristic morphology and the PCR made the diagnosis unequivocal. However, as the baby cleared the fever and infection without any trypanocidal drugs, a causal association between the parasite and the febrile illness is difficult to establish.

Theoretically, infection of humans with animal trypanosomes cannot occur because of a trypanolytic factor apolipoprotein L1 present in human serum [1]. Human infection with $T$. evansi was first reported by Joshi, et al. [3] in an immunocompetent adult from rural Nagpur, India. This patient was successfully treated with suramin, a drug used for treatment of human African trypanosomiasis [3]. Authors hypothesized that the patient was infected through a wound in the index finger while delivering infected cattle or through bite by Tabanidstriatus flies that transmit infection in animals. Subsequent to this case, a serologic study was conducted in the same village that showed that the sera of 81 of 1806 people $(4.5 \%)$ were seropositive for $T$. evansi infection by the card agglutination test but none had parasitemia on peripheral blood [4].

In India, infection with $T$. lewisi has been reported more frequently than T.evansi $[1,2,5-7]$. It is postulated that infection is transmitted to humans from infected rats through bite of rat fleas or contamination of open wounds with rat feces. Shrivastava, et al. [5] in 1974 reported two cases in adults from Raipur who recovered spontaneously [5]. Shah, et al. [7] reported a 6-week-girl living in a rat-infested flat in urban Mumbai who presented with fever, hepatosplenomegaly, anemia, thrombocytopenia and hepatitis; and trypanosomes morphologically resembling $T$. lewisi were detected in blood. She recovered spontaneously over a 2 week period. Verma, et al. [2] reported a case of a 37-day-old infant from Uttar Pradesh who presented with fever, lethargy and convulsions, and who had a history of painful insect bite the day before admission [2]. Treatment was initiated with ceftriaxone (for presumed sepsis) and liposomal amphotericin B (since it works for Leishmania), and later pentamidine. The parasitemia disappeared on day 7 of admission ( 2 days after starting pentamidine).

Our index case and those reported earlier probably reflect the tip of the iceberg of this possibly self-resolving infection. Nonetheless, it is important to increase awareness of this entity to ensure early diagnosis, avoidance of potentially toxic anti-trypansomal therapy in stable and improving patients, and more importantly to detect any change in disease epidemiology of this rare zoonotic infection.

Acknowledgements: Kirit Naik and Frenil Munim for their contribution in clinical care of the infant.

Contributors:DB: pediatrician incharge of clinical care and preparing the case summary; TS: opined on the strategy for management and prepared the manuscript. GSK: pathologist who first identified the trypansoma on the peripheral smear and participated in preparing the manuscript. PB and RG: identified the parasite as T. lewisi on basis of morphology and by PCR, and provided critical inputs to the preparation of the manuscript. Funding:None Stated; Competing interest: None.

\section{REFERENCES}

1. Joshi PP. Human trypanosomiasis in India: is it an emerging new zoonosis? In: Munjal YP, Editor, API Textbook of Medicine. $9^{\text {th }}$ edition. New Delhi:Jaypee Brothers; 2013.p.10-13.

2. Verma A, Manchanda S, Kumar N, Sharma A, Goel M, Banerjee PS, et al. Trypanosoma lewisi or T. lewisi-like infection in a 37-day-old Indian infant. Am J Trop Med Hyg. 2011;85:221-4.

3. Joshi PP, Shegokar VR, Powar RM, Herder S, Katti R, Salkar HR, et al. Human trypanosomiasis caused by Trypanosoma evansi in India: the first case report. Am J Trop Med Hyg.2005;73:491-5.

4. Shegokar VR, Powar RM, Joshi PP, Bhargava A, Dani VS, Katti R, et al. Human trypanosomiasis caused by Trypanosoma evansi in a village in India: preliminary serologic survey of the local population. Am J Trop Med Hyg. 2006;75:869-70.

5. Shrivastava KK, Shrivastava GP. Two cases of Trypanosoma (Herpetosoma) species infection of man in India. Trans R Soc Trop Med Hyg. 1974;68:3-4.

6. Doke PP,Kar A. A fatal case of Trypanosoma lewisi in Maharashtra, India. Ann Trop Med Public Health. 2011;4:91-5.

7. Shah I, Ali US, Andankar P, Joshi RR. Trypanosomiasis in an infant from India. J Vector Borne Dis. 2011;48:122-3. 